\title{
Les endothélines
}

La famille des endothélines comprend trois peptides de 21 acides aminés qui, injectés par voie intraveineuse chez le rat, provoquent une vasoconstriction de longue durée, précédée d'un épisode de dépression initiale. Ces peptides (ET-1, ET-2 et ET-3) sont codés par trois gènes différents et dérivent de précurseurs clivés par une enzyme de conversion spécifique. Deux récepteurs ont été clonés à ce jour, ET, qui lie ET-1 avec une plus forte affinité que ET-3 et relaie la vasoconstriction, et $\mathbf{E T}_{\text {в }}$ qui lie les endothélines avec la même et forte affinité et relaie la vasodilatation. Les endothélines exercent aussi des effets non vasculaires dans le cour, le rein, le cerveau et le foie, où elles se conduisent en neuropeptides et facteurs de croissance. Les voies et intermédiaires de la transmission des signaux issus de la liaison des endothélines à leurs récepteurs sont nombreux et complexes, mettant en jeu différents types de seconds messagers $\left(\mathrm{Ca}^{2+}\right.$, AMP cyclique...). Le développement de nombreux agonistes, antagonistes et inhibiteurs des enzymes de conversion permet d'améliorer la connaissance des effets de ces hormones et pourrait aboutir à des médicaments actifs dans différentes situations pathologiques, notamment le choc cardiovasculaire.

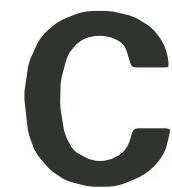
ing ans à peine se sont écoulés depuis la découverte de l'endothéline-1, le plus puissant peptide vasoconstricteur connu à ce jour, et l'on ne compte plus les publications relatives à ses effets vasculaires et non vasculaires. L'histoire commence en 1985, quand Hickey et al. décrivent que l'addition d'un milieu de culture de cellules endothé- liales d'aorte bovine à des sections d'artères coronaires provoque une vasoconstriction de longue durée [1]. En 1988, M. Yanagisawa et ses collaborateurs, du laboratoire de T. Masaki (Tsukuba, Japon) ont isolé et cloné ce facteur vasoconstricteur, un peptide de 21 acides aminés, qu'ils ont nommé endothéline-1 [2]. Deux autres formes de ce peptide, l'endothéline-2 (et son équivalent 
murin, l'endothéline $\beta$ ou vasointestinal contractor) et l'endothéline-3 ont ensuite été mises en évidence par ce même groupe. Elles sont également constituées de 21 acides aminés, mais leur structure diffère de celle de l'endothéline-1 par deux et six acides aminés respectivement [3]. En parallèle, une équipe israélienne dirigée par M. Sokolovski a isolé des toxines de venin d'aspic, les sarafotoxines, qui possèdent une structure similaire à celle des endothélines, suggérant une origine évolutive commune de ces deux familles de peptides [4]. Les sarafotoxines et les endothélines ont en commun une structure comportant deux ponts disulfure situés entre les cystéines en position 1 et 15 d'une part, et 3 et 11 d'autre part, et une extrémité C-terminale identique (figure 1).

\section{Biosynthèse et dégradation}

Les trois formes d'endothélines sont libérées à partir de trois précurseurs différents, de 200 acides aminés chacun, les préproendothélines, chacune codée par un gène différent [3, 4]. Les préproendothélines sont ensuite clivées par coupure protéolytique à des sites d'acides aminés dibasiques (Lys-Arg et Arg-Arg) en proendothélines (ou big endothelins) de 37, 38 ou 41 acides aminés (big ET-2, big ET-1 humaine ou big ET-3, respectivement). Les big endothelins sont mûries en endothélines par clivage à un site tout à fait inhabituel, Trp-Val pour l'ET-1 et l'ET-2 ou Trp-Ile pour l'ET-3 (figure 2), par des enzymes spécifiques à chaque forme, les endothelin converting enzymes ou ECE. On ne possède aucune donnée sur les ECE-2 et-3, en revanche l'ECE-1 a été purifiée et correspond à une nou-

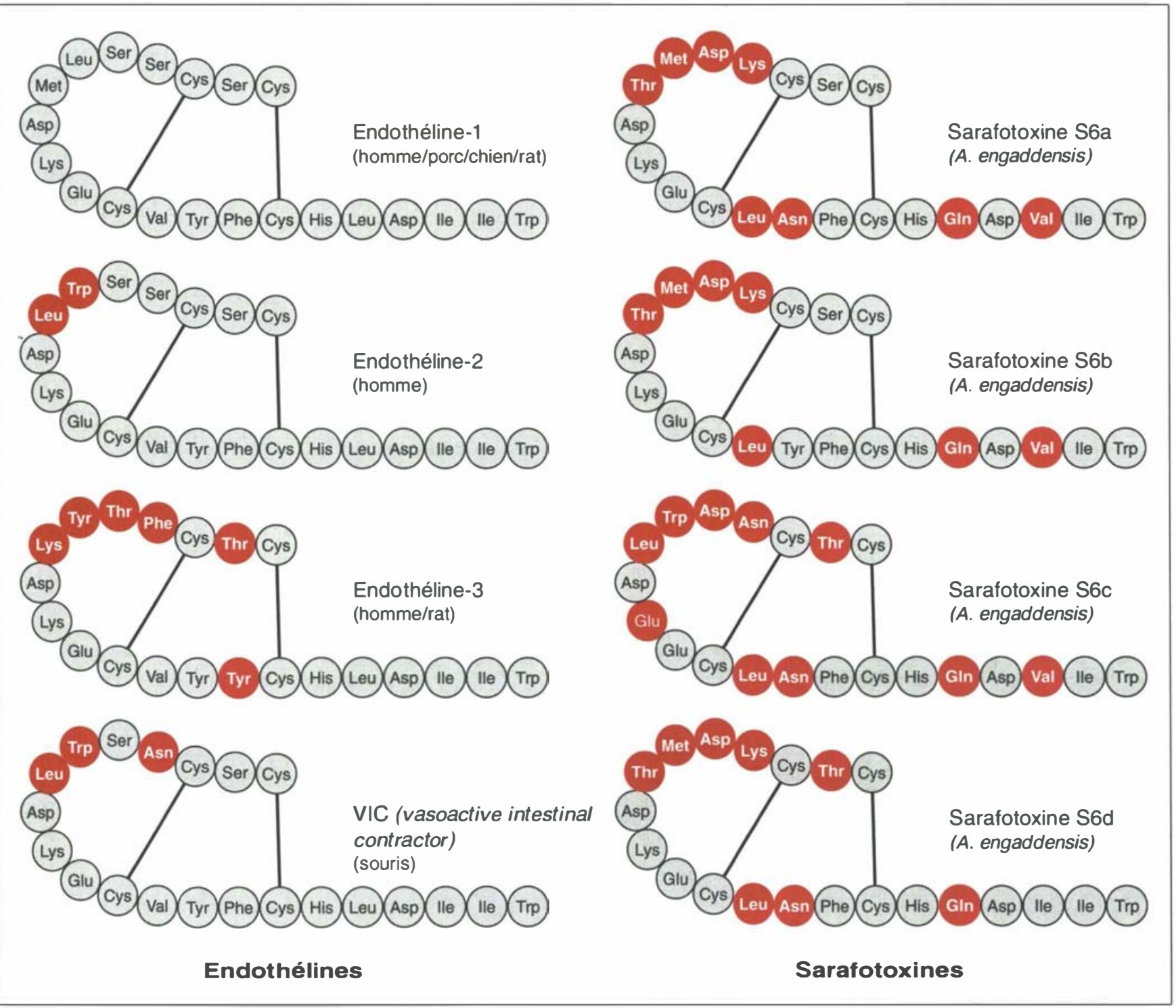

Figure 1. Structure des endothélines et des sarafotoxines. En rouge sont indiqués les acides aminés qui diffèrent de ceux de l'ET-1. 


\section{RÉFÉRENCES}

1. Hickey K, Rubanyi G, Paul R, Highsmith R. Characterization of a coronary vasoconstrictor produced by endothelial cclls in culture. Am J Physiol 1985 ; 248 : C550-6.

2. Yanagisawa M, Kurihara $H$, Kumura $S$, et al. A novel potent vasoconstrictor peptide produced by vascular endothelial cells. Nature 1988 ; 332: 411-5.

3. Sakurai T, Yanagisawa M, Masaki T. Molecular characterization of endothelin receptors. Trends Pharmacol Si $1992 ; 13$ : 103-8.

4. Sokolovsky M. Endothelins and sarafotoxins: physiological regulation, receptor subtypes and transmembrane signaling. Pharmacol Ther 1992; 54: 129-49.

5. Boulanger C, Lüscher T. Release of endothelin from the porcine aorta. Inhibition by endothelium-derived nitric oxide. $J$ Clin Invest $1990 ; 85$ : 587-90.

6. Kohno M, Yasunari K, Yokokawa K, Murakawa K, Horio T, Takeda T. Inhibition by atrial and brain natriuretic peptides of endothelin-1 secretion after stimulation with angiotensin II and thrombin of cultured human endothelial cells. J Clin Invest 1991; 87 : 1999-2004.

7. Ming $\mathrm{Hu} \mathrm{R}$, Levin ER, Pedram A, Frank HJL. Atrial natriuretic peptide inhibits the production and secretion of endothelin from cultured endothelial cells. $J$ Biol Chem 1992; 267 : 17384-9.

8. Sakamoto A, Yanagisawa M, Sawamura $\mathrm{T}$, et al. Distinct subdomains of human endothelin receptors determine their selectivity to endothelin $\mathrm{n}_{\mathrm{A}}$-selective antagonist and endothelin ${ }_{\mathrm{B}}$-selective agonists. $J$ Biol Chem 1993; 268: 8547-53.

9. Shetty SS, Okada T, Webb RL, Del Grande D, Lappe RW. Functionally distinct endothelin B receptors in vascular endothelium and smooth muscle. Biochem Biophys Res Commun 1993 ; 191 : 459-64.

10. Clozel M, Gray GA, Breu V, Löffler $\mathrm{BM}$, Osterwalder R. The endothelin $\mathrm{ET}_{\mathrm{B}}$ receptor mediates both vasodilation and vasoconstriction in vivo. Biochem Biophys Res Commun $1992 ; 186$ : 867-73.

11. Sokolovsky M, Ambar I, Galron R. A novel subtype of endothelin receptors. $J$ Biol velle forme de métalloprotéase, sensible au phosphoramidon mais insensible à certains autres inhibiteurs classiques de métalloprotéases (captocril ou kélatorphan) $[3,4]$. Des quantités équimolaires de big ET-1 et ET-1 sont retrouvées dans le plasma mais, in vitro, la big ET-1 possède un effet vasoconstricteur 100 fois plus faible que celui de l'ET-1, suggérant que l'étape de conversion de la big ET-1 est importante pour l'activité biologique de l'ET-1.

La dégradation des endothélines est assurée en deux étapes par une endopeptidase neutre (NEP) sensible au phosphoramidon. L'ET-1 est tout d'abord clivée au site Ser6-Leu7, produisant un peptide encore actif biologiquement, puis l'ET-1 est inactivée par clivage entre Asp18 et Ile19. L'ET-2 et l'ET-3 seraient clivées en une étape entre Asp18 et Ile19. Les sarafotoxines sont beaucoup plus résistantes que les endothélines à la dégradation par la NEP, ce qui pourrait expliquer leur très forte toxicité [4].

\section{Les endothélines sont des "hormones locales"}

La demi-vie de l'endothéline-1 dans le plasma est très courte $(<1 \mathrm{~min})$ et sa concentration circulante $(0,1$ à 5 pM suivant les auteurs) est bien inférieure aux concentrations généralement nécessaires à l'obtention de ses effets biologiques. De plus, les ARN messagers codant pour les préproET-1 (2,3 kb) et prépro-ET-3 (3,7 kb) sont exprimés (en proportions différentes) non seulement dans les tissus vasculaires mais également dans le poumon, le cerveau, le rein, le cceur, l'estomac, le pancréas, le testicule, la rate, etc. [3, 4] Toutefois, seul l'ARN messager codant pour la prépro-ET-1 est exprimé dans les cellules endothéliales vasculaires. L'ET-2 est présente dans le côlon, le cceur, l'intestin, ainsi que dans la médullaire rénale. Quant à la localisation cellulaire de synthèse de l'ET-1, il est maintenant établi qu'elle n'est pas limitée aux cellules endothéliales, mais s'étend également aux cellules épithéliales et aux macrophages [3, 4].

Différents agents stimulent la transcription des gènes codant pour les endothélines, en particulier les esters de phorbol, la thrombine, l'insuline, l'endotoxine, certains vasoconstricteurs (vasopressine, angiotensine II, adrénaline), ou des facteurs de croissance (transforming growth factor $\beta 1$, platelet-derived growth factor, epidermal growth factor) [3, 4]. La sécrétion d'endothéline est également contrôlée par les agents vasodilatateurs, le monoxyde d'azote (NO ou EDRF) ou le facteur natriurétique (ANF). Le monoxyde d'azote, formé à partir de la L-arginine, active la guanylate cyclase soluble et augmente donc la concentration intracellulaire de GMP cyclique. Boulanger et Lüscher ont montré que la production aortique d'endothéline (ET-1) était potentialisée par des inhibiteurs de guanylate cyclase soluble, alors que l'addition de GMP cyclique bloquait cette production [5]. L'ANF inhibe la sécrétion d'ET-1 par l'intermédiaire de deux récepteurs différents dans les cellules endothéliales d'aorte et de veine ombilicale, le récepteur $B$ contenant la guanylate cyclase membranaire, et le récepteur $\mathrm{C}$ dont l'activation aboutit respectivement à une augmentation de GMP cyclique [6] et à une inhibition de la production d'AMP cyclique [7].

\section{Hétérogénéité des récepteurs des endothélines}

L'injection intraveineuse d' ET-1, d'ET-2 ou d'ET-3 chez le rat conduit tout d'abord à une diminution de la pression artérielle, puis à une hypertension prolongée. Ces deux phases seraient relayées par des mécanismes différents, l'ET-1 étant un vasoconstricteur plus puissant que l'ET-3 alors que les deux peptides possèdent des activités hypotensives équivalentes. De même, l'ET-1 perfusée dans l'artère mésentérique provoque un effet vasoconstricteur plus puissant que celui observé au cours de la perfusion d'ET-3, alors qu'à faibles doses, les deux peptides induisent un effet vasodilatateur comparable. Cet effet vasodilatateur serait lié au relargage par les endothélines de substances vasodilatatrices dérivées de l'endothélium $[3,4]$. Sur la base de ces données, il avait été suggéré que les effets vasculaires des endothélines étaient relayés par deux classes de 
récepteurs, l'une $\left(\mathrm{ET}_{\mathrm{A}}\right)$ relayant les effets vasoconstricteurs alors que l'autre $\left(\mathrm{ET}_{\mathrm{B}}\right)$ conduirait à la production de facteurs relaxants (monoxyde d'azote, prostacycline $\mathrm{PGI}_{2}$ ) et à la vasodilatation (figure 3). Ces observations ont été confirmées sur la base de données biochimiques qui ont permis d'établir que le récepteur présent dans le muscle lisse vasculaire lie l'ET-1 avec une affinité très supérieure à l'ET-3, alors que le récepteur présent dans les cellules endothéliales vasculaires lie les deux peptides avec la même affinité. Ces deux classes de site de liaison des endothélines ont été identifiées par des études de liaison d'125I-ET-1 dans de nombreux tissus (rein, poumon, cerveau, foie...) [3, 4]. Récemment, des ligands synthétiques spécifiques de chaque type de récepteurs ont été synthétisés. Ainsi, le BQ 123, un pentapeptide cyclique (cyclo-(DTrp-D-Asp-Pro-D-Val-Leu) et le $B Q 153$, l'un de ses dérivés, sont des antagonistes sélectifs des récepteurs $\mathrm{ET}_{\mathrm{A}}$. Il n'existe pas actuellement d'antagoniste des récepteurs $\mathrm{ET}_{\mathrm{B}}$, mais, en revanche, différents agonistes spécifiques ont été synthétisés, le BQ3020 ( $\mathrm{N}$-acetyl$\left(\mathrm{Ala}^{11,15}\right)$ ET-1 (6-21) et l'IRL1620 (N-succinyl-(glu ${ }^{9}, \quad\left(\mathrm{Ala}^{11,15}\right)$ ET-1 (8-21). La sarafotoxine-S6C est également largement utilisée en tant qu'agoniste sélectif de ces récepteurs $[3,4]$.

Deux classes de récepteurs des ET ont été clonées chez le bœuf, le rat et l'homme. L'ADN complémentaire cloné du poumon de bœuf, transfecté dans des cellules de mammifères COS dépourvues de récepteurs des endothélines, a conduit à l'expression d'un récepteur qui lie avec la même affinité les trois formes d'endothéline et la sarafotoxine S6B [3, 4]. L'ARN messager codant pour ce récepteur ( récepteur $\mathrm{ET}_{\mathrm{B}}$ ) est présent dans un grand nombre de tissus, mais n'est pas exprimé dans les cellules musculaires lisses d'origine vasculaire. En revanche, l'ADN complémentaire cloné du poumon de rat, transfecté dans les mêmes cellules COS, exprime un récepteur liant spécifiquement l'ET-1. L'ARN messager codant pour ce récepteur (récepteur $\mathrm{m} / \mathrm{s} n^{\circ} 10 \mathrm{vol}$. 9, oclobre 93

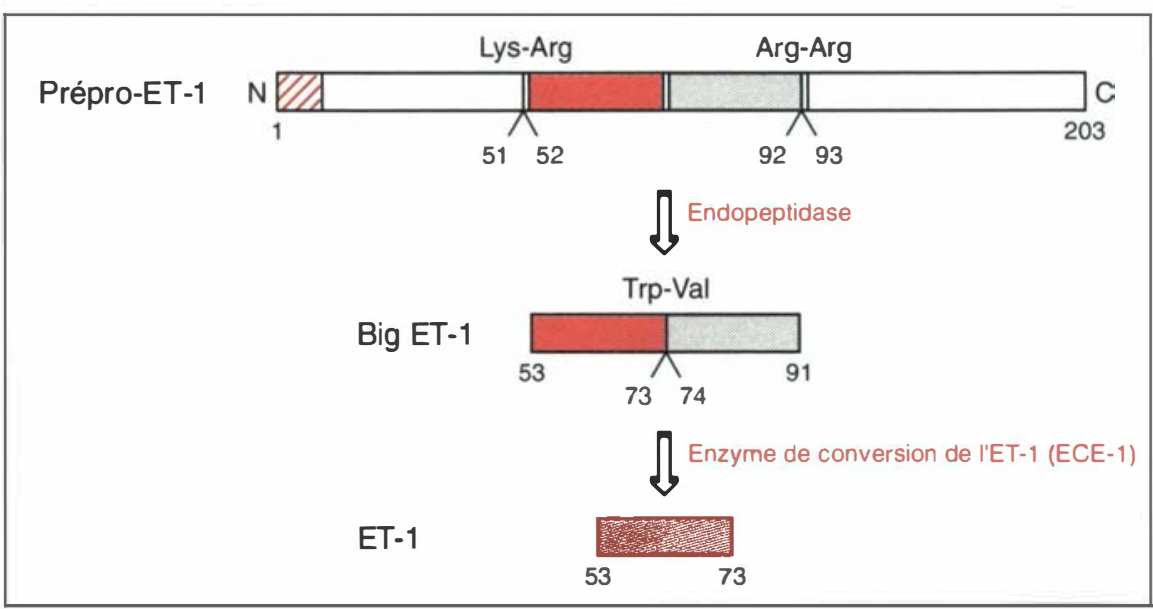

Figure 2. Maturation post-traductionnelle de I'ET-1. Les acides aminés sont numérotés à partir de leur position dans la prépro-ET-1.

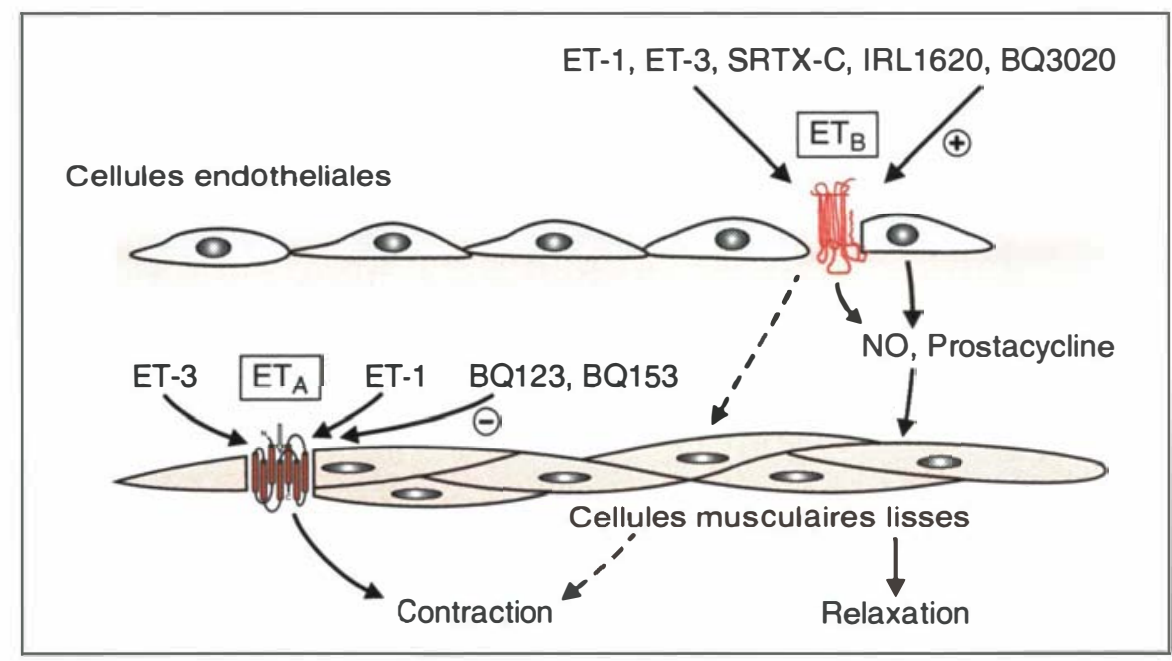

Figure 3. Effets vasodilatateurs et vasoconstricteurs des endothélines. Le récepteur $E T_{A}$, présent dans les cellules musculaires lisses, possède une forte affinité pour I'ET-1 et une faible affinité pour I'ET-3. Le BO 123 et le BO 153 sont des antagonistes du récepteur $E T_{A}$. Ce récepteur relaie les effets vasoconstricteurs des endothélines. Le récepteur $E T_{B}$, présent dans les cellules endothéliales, possède la même affinité pour I'ET-1 et I'ET-3. La sarafotoxine S6C (SRTX-C), I'IRL 1620 et le BQ 3020 sont des agonistes spécifiques des récepteurs $E T_{B}$. Ce récepteur relaie les effets vasodilatateurs des endothélines par l'intermédiaire du monoxyde d'azote ou des prostacyclines. Cependant, des données récentes indiquent qu'une sous-classe de récepteurs $E T_{B}$ pourrait également relayer certains effets vasoconstricteurs des endothélines (voir texte). 


\section{RÉFÉRENCES}

12. Goto K, Kasuya Y, Matsuki N, et al Endothelin activates the dihydropyridinesensitive, voltage-dependent $\mathrm{Ca}^{2}+$ channel in vascular smooth muscle. Proc Natl Acad Sci USA 1989 ; 86 : 3915-8.

13. Jouneaux C, Goldsmith P, Hanoune J, Lotersztajn S. Endothelin inhibits the calcium pump and stimulates phosphoinositide phospholipase $\mathrm{C}$ in liver plasma membranes via two different $\mathrm{G}$ proteins, Gs and $\mathrm{Gq}$ J Cardiovascular Pharmacol 1993 (sous presse)

14. Takuwa N, Takuwa $\mathrm{Y}$, Yanagisawa $\mathrm{M}$, Yamashita K, Masaki T. A novel vasoactive peptide endothelin stimulates mitogenesis through inositol lipid turnover in Swiss 3T3 fibroblasts. J Biol Chem 1989; 264 7856-61.

15. Aramori I, Nakanishi S. Coupling of two endothelin receptor subtypes to differing signal transduction in transfected chinese hamster ovary cells. J Biol Chem $1992 ; 267$ : 12468-74.

16. Eguchi S, Hirata Y, Imai T, Marumo F. Endothelin receptor subtypes are coupled to adenylate cyclase via different guanyl nucleotide-binding proteins in vasculature. Endocrinology $1993 ; 132$ : 524-9.

17. Force T, Kyriakis JM, Avruch J, Bonventre JV. Endothelin, vasopressin, and angiotensin II enhance tyrosine phosphorylation by protein kinase C-dependent and independent pathways in glomerular mesangial cells. J Biol Chem 1991 ; 266 : 6650-6.

18. Masaki T. Endothelins: homeostatic and compensatory actions in the circulatory and endocrine systems. Endocrine Rev 1993 ; 14 : 256-68.

19. Battistini B, Chailler P, D'Orlćans-Juste $P$, Bric̀re $N$, Sirois $P$. Growth regulatory properties of endothelins. Peptides $1993 ; 14$ : 385-99

20. Simonson MS, Herman WH. Protein kinase $\mathrm{C}$ and protein tyrosine kinase activity contribute to mitogenic signaling by endothelin-1. J Biol Chem 1993; 268 : 9347-57.

21. Wang Y, Simonson MS, Pouyssćgur J, Dunn MJ. Endothelin rapidly stimulates mitogen-activated protein kinase activity in rat mesangial cells. Biochem J $1992 ; 287$ : 589-94.

22. Serradeil-Le Gal C, Jouneaux C, Sanchez-Bueno A, et al. Endothelin action in rat liver. Receptors, free $\mathrm{Ca}^{2+}$ oscillations, and activation of glycogenolysis. J Clin Invest $1991 ; 87$ : 133-8.

23. Gandhi C, Stephenson K, Olson M Endothelin, a potent peptide agonist in the liver. J Biol Chem 1990; 265 : 17432-5.

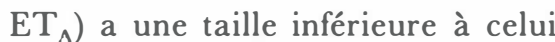
du récepteur $E T_{B}$, est également présent dans de nombreux tissus, et prédomine dans les muscles lisses d'origine vasculaire [3, 4]. Les gènes codant pour ces récepteurs ont été clonés chez l'homme et sont localisés sur les chromosomes 13 $\left(\mathrm{ET}_{\mathrm{A}}\right)$ et $4\left(\mathrm{ET}_{\mathrm{B}}\right)$. Les récepteurs $E T_{A}$ et $E_{B} T_{B}$ sont couplés aux protéines $G$, et possèdent une structure comprenant sept domaines transmembranaires, une extrémité $\mathrm{N}$ terminale extraccllulaire et une extrémité C-terminale cytoplasmique. Ils présentent entre eux une homologie de séquences en acides aminés de $60 \%$ et divergent essentiellement dans la partie $\mathrm{N}$ terminale de la molécule, aussi bien dans la composition en acides aminés que dans la longucur de la portion $\mathrm{N}$-terminale du réccpteur (80 résidus pour le récepteur $\mathrm{ET}_{\wedge}$ et 101 pour le récepteur $\mathrm{ET}_{\mathrm{B}}$, et ce, quelle que soit l'espèce considérée) $[3,4]$. L'utilisation de récepteurs chimériques, construits en substituant progressivement la structure du récepteur $E T_{A}$ humain avec celle de son homologue $\mathrm{ET}_{\mathrm{B}}$ a permis récemment de déterminer que des portions différentes des récepteurs sont impliquées dans la sélectivité pour les agonistes et les antagonistes [8]. Ainsi, la région $\mathrm{N}$ terminale de l'ET-1 (variable) et sa partic C-terminale (commune) seraient nécessaires à la liaison, interagissant respectivement avec les domaines transmembranaires 4, 5, 6 et $1,2,3,7$. En accord avec ces données, le BQ123, qui pourrait mimer la portion C-terminale de l'ET-1, sc lierait aux domaines 1-3, 7. En revanche, seulc l'extrémité Cterminale des ET serait requise pour l'interaction des ET avec le récepteur $\mathrm{ET}_{\mathrm{B}}$, interaction se produisant avec les domaines $1,2,3$, $4,5,6$ et 7 du réceptcur. De façon surprenante, les extrémités $\mathrm{N}$ terminales des récepteurs $\mathrm{ET}_{\mathrm{A}}$ et $\mathrm{ET}_{\mathrm{B}}$, remarquables par leur différence, ne jouent aucun rôle dans la sélectivité pour le ligand [8].

Il existe probablement d'autres classes de récepteurs des endothélines. Alors que classiquement les récepteurs $\mathrm{ET}_{\mathrm{B}}$ relayaient uniquement les effets vasodilatateurs des endo- thélines, ils apparaissent aujourd'hui également impliqués dans certains effets vasoconstricteurs. Ainsi, l'IRL 1620, un agoniste spécifique des récepteurs $\mathrm{ET}_{\mathrm{B}}$, est capable de provoquer des contractions de l'artère coronaire, même en présence de $B Q 123$, un antagoniste des réceptcurs $\mathrm{ET}_{\text {19]. De même, les }}$ contractions de la veine saphène provoquées par la sarafotoxine S6C, l'agoniste spécifique des récepteurs $\mathrm{ET}_{\mathrm{B}}$, sont insensibles au $B Q 123[10]$. Ces données suggèrent l'existence de deux sous-classes de réceptcurs $E_{\mathrm{B}}$, l'une relayant la vasodilatation, l'autre la vasoconstriction de certaines artères ou veines. En accord avec cette hypothèse, Sokolovsky et al. ont récemment caractérisé deux sous-types de récepteurs $\mathrm{ET}_{\mathrm{B}}$, l'un de très forte affinité (pM) qui pourrait relayer les effets vasodilatateurs, l'autre de forte affinité (nM) qui pourrait être responsable des effets vasoconstricteurs [11]. D'autre part, un récepteur liant l'ET-3 avec plus d'affinité que l'ET-1 (récepteur $\mathrm{ET}_{\mathrm{C}}$ ) a été caractérisé dans le poumon, l'aorte et le cœur, mais les effets biologiques associés à ce récepteur restent à déterminer. Le fait que, même à faible stringence, les sondes $\mathrm{ADNc}$ codant pour les récepteurs $\mathrm{ET}_{\wedge}$ et $\mathrm{ET}_{\mathrm{B}}$ hybrident uniquement avec deux fragments d'ADN génomique (correspondant aux gènes des récepteurs $E_{\wedge}$ et $\mathrm{ET}_{\mathrm{B}}$, respectivement) suggère que le gène codant pour un éventuel troisième récepteur doit présenter peu d'homologie de séquence avec les deux classes de récepteurs déjà clonés $[3,4]$.

\section{Les seconds messagers des endothélines}

Il est clair aujourd'hui que les endothélines exercent leurs effets biologiques en empruntant différentes voies de transduction (Tableau I).

Dans tous les tissus, la liaison de l'ET-1 aux récepteurs $\mathrm{ET}_{\wedge}$ et $\mathrm{ET}_{\mathrm{B}}$ provoque une augmentation du taux de calcium cytosolique [3, 4]. Comme pour les autres hormones mobilisant le calcium, cette augmentation est la conséquence de l'activation conjointe d'au moins 
deux systèmes membranaires, la phospholipase $\mathrm{C}$ et l'influx de calcium à travers des canaux calciques. La stimulation de la phospholipase C par les endothélines conduit à l'apparition de deux seconds messagers, l'inositol 1,4,5 triphosphate (IP3) soluble, et le diacyglycérol, une molécule qui reste dans la membrane plasmique et active la protéine kinase C. L'IP3 se lie à des récepteurs-canaux calciques dans le réticulum endoplasmique/sarcoplasmique, et la sortie de calcium qui en résulte est extrêmement rapide. Deux classes de protéines $G$ couplent les récepteurs des ET à la phospholipase C. Suivant les tissus, des protéines $G$ sensibles ou insensibles à la toxine de Bordetella pertussis ont été impliquées [3, 4]. Le maintien de la réponse cellulaire est assuré par une augmentation de l'influx de calcium à travers des canaux calciques de la membrane plasmique [3, 4]. Les premières observations publiées par l'équipe de Masaki [12] laissaient supposer que, dans les muscles lisses vasculaires, l'ET-1 était un agoniste des canaux calciques dépendants du voltage de type L. Cependant, aujourd'hui, de nombreuses données démontrent que l'influx de calcium provoqué par l'ET-1 est observé en présence d'inhibiteurs de ces canaux calciques $\mathrm{L}$, et que d'autres canaux, les receptor-operated calcium channels (ROC), seraient également cibles des endothélines [3, 4]. L'identité de ces canaux calciques et le mécanisme déclenchant leur ouverture restent donc à déterminer et dépendent probablement du tissu considéré. Nous avons également observé que l'ET-1 inhibe la pompe à calcium des membranes plasmiques de foie, par l'intermédiaire d'une protéine Gs, bloquant ainsi l'extrusion de calcium hors de la cellule [13]. Ce système contribuerait donc également au signal calcique activé par les endothélines. L'incubation de cellules mésangiales ou de fibroblastes avec des esters de phorbol, activateurs de protéine

\section{Tableau 1}

LES DIFFÉRENTES VOIES DE TRANSDUCTION DES ENDOTHÉLINES

\begin{tabular}{|c|c|c|c|c|}
\hline Récepteur & Protéine G & Effecteur & Effet & Second messager \\
\hline $\mathrm{ET}_{A} / \mathrm{ET}_{B}$ & $\mathrm{Gi} / \mathrm{Gq}$ & $\begin{array}{c}\text { PLCB } \\
\text { (IP3, DAG) }\end{array}$ & + & $/ \mathrm{Ca}^{2+} / \mathrm{PKC}$ \\
\hline$?$ & $\stackrel{?}{\mathrm{Gi}}$ & $\begin{array}{c}\text { Canal } \mathrm{Ca}^{2+} \\
\text { ROC } \\
\text { canal L }\end{array}$ & + & $/ \mathrm{Ca}^{2+}$ \\
\hline $\mathrm{ET}_{\mathrm{B}}$ & Gs & $\begin{array}{l}\text { Pompe à } \mathrm{Ca}^{2+} \\
\text { des membranes } \\
\text { plasmiques }\end{array}$ & - & $/ \mathrm{Ca}^{2+}$ \\
\hline$?$ & ? & $\begin{array}{c}\mathrm{PLC}_{\gamma} \\
\text { (phosphorylation } \\
\text { tyrosine) }\end{array}$ & + & $/ \mathrm{Ca}^{2+} / \mathrm{PKC} \ldots$ \\
\hline $\mathrm{ET}_{\mathrm{A}}$ & Gs & $\begin{array}{l}\text { Adénylate } \\
\text { cyclase }\end{array}$ & + & /AMPc \\
\hline $\mathrm{ET}_{\mathrm{B}}$ & Gi & $\begin{array}{l}\text { Adénylate } \\
\text { cyclase }\end{array}$ & - & $\backslash \mathrm{AMPC}$ \\
\hline $\mathrm{ET}_{\mathrm{A}}$ & $?$ & PLD & + & $\begin{array}{c}\text { hydrolyse } \\
\text { phosphatidylcholine } \\
\text { (/ PKC) }\end{array}$ \\
\hline$?$ & $?$ & $\mathrm{PLA}_{2}$ & + & $\begin{array}{l}\text { Acide } \\
\text { arachidonique }\end{array}$ \\
\hline $\mathrm{ET}_{\mathrm{B}}$ & & $\mathrm{Na}^{+} / \mathrm{H}^{+}$ & + & $/ \mathrm{pHi}$ \\
\hline
\end{tabular}

PLC, PLD, PLA = phospholipases $\mathrm{C}, \mathrm{D}, \mathrm{A} ; \mathrm{IP3}=$ inositol triphosphate $; \mathrm{DAG}=$ diacylglycérol $; \mathrm{ROC}=$ receptor-operated calcium channels $; \mathrm{PKC}=$ protéine kinase $C$; $\mathrm{pHi}=\mathrm{pH}$ intracellulaire.

$\mathrm{m} / \mathrm{s} n^{\circ} 10$ vol. 9, octobre 93 kinase $\mathrm{C}$, atténue l'augmentation de calcium intracellulaire provoquée par l'ET-1 [14]. C'est donc par l'intermédiaire de la protéine kinase C que, par un mécanisme de feedback négatif, pourrait être contrôlé le signal calcique stimulé par l'endothéline. D'autre part, l'activation de la protéine kinase $\mathrm{C}$ par les endothélines semble être un élément important dans l'initiation de la réponse proliférative des cellules aux endothélines.

Ce n'est que très récemment que des variations d'AMP cyclique ont été mises en évidence sous influence des endothélines. L'expression des récepteurs $\mathrm{ET}_{\mathrm{A}}$ et $\mathrm{ET}_{\mathrm{B}}$ dans des cellules $\mathrm{CHO}$ a permis de montrer que l'ET-1 stimule la production d'AMP cyclique dans les cellules exprimant le récepteur $\mathrm{ET}_{\mathrm{A}}$, alors qu'elle l'inhibe dans des cellules tranfectées avec les récepteurs $\mathrm{ET}_{\mathrm{B}}[15]$. Ces résultats ont été confirmés par Eguchi et al. qui ont observé que dans des cellules musculaires lisses d'origine vasculaire n'exprimant que le récepteur $\mathrm{ET}_{\mathrm{A}}$, l'ET-1 stimule la production d'AMP cyclique, alors que dans les cellules endothéliales vasculaires, qui ne possèdent que le récepteur $\mathrm{ET}_{\mathrm{B}}$, l'ET-1 inhibe la production d'AMP cyclique [16]. L'activation et l'inhibition de la production d'AMP cyclique dans ces cellules sont relayées par les protéines $\mathrm{Gs}$ et $\mathrm{Gi}$ respectivement.

Bien que les récepteurs des ET ne possèdent pas d'activité tyrosine kinase intrinsèque, ces peptides stimulent la phosphorylation de plusieurs protéines cytosoliques sur des résidus tyrosine par deux mécanismes distincts, dépendants et indépendants de la protéine kinase C [17]. L'identité de ces protéines n'est pas définie ; cependant l'une d'entre elles pourrait être l'isoforme $\gamma 1$ de la phospholipase $\mathrm{C}$, isoforme généralement couplée aux facteurs de croissance [17]. Egalement, il est important de noter que certaines protéines phosphorylées sur des résidus tyrosine par l'ET-1 le sont également par l'EGF [17], suggérant l'existence d'intermédiaires communs entre ces deux classes de récepteurs, l'un couplé aux protéines $G$, l'autre comportant une activité tyrosine kinase intrinsèque. 


\section{RÉFÉRENCES}

24. Jouneaux C, Serradeil-Le Gal C, Raufaste, et al. Biological effects of sarafotoxin in rat liver. Biochem Pharmacol $1992 ; 43$ : 1146-8.

25. Gandhi C, Stephenson K, Olson M. A comparative study of endothelin- and platelet-activating-factor-mediated signal transduction and prostaglandin synthesis in rat Kupffer cells. Biochem J 1992 ; 281 : 485-92.

26. Pinzani M, Failli P, Ruocco C, et al. Fat-storing cells as liver-specific pericytes. $J$ Clin Invest 1992 ; 90 : 642-6.

27. Kawada N, Tran-Thi TA, Klein H, Decker K. The contraction of hepatic stellate (Ito) cells stimulated with vasoactive substances : possible involvement of endothelin 1 and nitric oxide in the regulation of the sinusoidal tonus. Eur J Biochem 1993 ; 213 : 815-23.

28. Oshita M, Takei Y, Kawano S, et al. Roles of endothelin-1 and nitric oxide in the mechanism for ethanol-induced vasoconstric tion in rat liver. $J$ Clin Invest $1993 ; 91$ : 1337-42

29. Stojilkovic SS, Catt KJ. Neuroendocrine actions of endothelins. Trends Pharmacol Sci $1992 ; 13$ : 385-91.

30. Krämer BK, Nishida M, Kelly RA, Smith TW. Endothelins. Myocardial actions of a new class of cytokines. Circulation 1992 ; 85 : 350-6.

31. Vigne P, Breittmayer JP, Marsault R, Frelin C. Endothelin mobilizes $\mathrm{Ca}^{2}+$ from a caffeine- and ryanodine-insensitive intracellular pool in rat atrial cells. $J$ Biol Chem $1990 ; 265$ : 6782-7.

32. Suzuki T, Hoshi H, Mitsui Y. Endothelin stimulates hypertrophy and contractility of neonatal rat cardiac myocytes in a serum-free medium. FEBS Lett 1990 ; 268 : 149-51.

33. Shirakami G, Nakao K, Saito Y, et al. Low doses of endothelin-1 inhibit atrial natriuretic peptide secretion. Endocrinology 1993 ; 132 : 1905-12.

34. Irons CE, Sei CA, Hidaka H, Glembotski CC. Protein kinase $\mathrm{C}$ and calmodulin kinase are required for endothelinstimulated atrial natriuretic factor secretion from primary atrial myocytes. $J$ Biol Chem $1992 ; 267: 5211-6$.

35. Webb DJ. Endothelin receptors cloned, endothelin converting enzyme characterized and pathophysiological roles for endothelin proposed. Trends Pharmacol Sci 1991; 12 : 43-6.

36. Warner TD, Vane JR. ET touches down in Houston. Nature 1993 ; 362 : 497-8.

37. Giaid A, Yanagisawa M, Langleben D, et al. Expression of endothelin-1 in the lungs of patients with pulmonary hypertension. $N$ Engl J Med 1993 ; 328 : 1732-9.
L'ET-1 active la phospholipase D, qui hydrolyse la phosphatidylcholine en acide phosphatidique, ce qui conduit à une production prolongée de diacylglycérol, indépendamment de toute production d'IP3 [4]. L'ET-1 stimule également la phospholipase A2 [4] et donc la production d'acide arachidonique, de thromboxane A2, de prostacycline et de prostaglandines. Cependant, la nature des eicosanoïdes produits dépend de l'espèce et du tissu. L'ET-1 stimule l'échangeur $\mathrm{Na}^{+} / \mathrm{H}^{+}$, conduisant à l'alcalinisation du $\mathrm{pH}$ cytosolique, une étape nécessaire à l'initiation de la prolifération cellulaire. Cette activation est, ou non, consécutive à l'activation de la phospholipase $\mathrm{C}$, et à la stimulation de la protéine kinase $\mathrm{C}$ qui en découle [4]. L'ET-1 stimule la $\mathrm{Na}^{+} / \mathrm{K}^{+}$ATPase dans le rein, secondairement à une augmentation des prostaglandines PGE2, et inhibe ce système dans l'aorte, par un mécanisme dépendant de la protéine kinase C [4]. Enfin, l'ET-1 module l'activité de différents canaux ioniques. Ainsi, elle ouvre un canal cationique non sélectif, inhibe certains canaux $\mathrm{K}^{+}$(courants lents sortants et canaux sensibles à l'ATP), et stimule le canal chlore [18].

\section{Effets biologiques des endothélines}

Très rapidement, la présence d'ARN messagers codant pour les endothélines et leurs récepteurs a été détectée dans différents tissus, suggérant que les endothélines pouvaient agir localement de façon autocrine ou paracrine au niveau des tissus cibles. En accord avec ces données, et bien que les endothélines aient été initialement mises en évidence pour leur puissants effets vasoconstricteurs que nous avons décrits précédemment (figure 3), de nombreux effets biologiques de ces peptides dans des tissus non vasculaires ont été décrits (Tableau II).

L'une des propriétés essentielles de l'endothéline-1 est son effet mitogène. Ainsi, les endothélines stimulent la prolifération des fibroblastes, des cellules musculaires lisses vasculaires, des cellules mésangiales, des mélanocytes, des cellules endothéliales de capillaires cérébraux, des péricytes vasculaires, ou des cellules gliales [19]. Cet effet s'exerce également sur différentes lignées de cellules tumorales en culture [19]. Le plus souvent, l'activité proliférative des endothélines est relayée par le récepteur $\mathrm{ET}_{\mathrm{A}}$. Comparée à celle d'autres facteurs de croissance (EGF, PDGF, etc.), l'activité mitogène des endothélines est faible, et souvent augmentée en présence d'autres agents mitogènes (insuline, EGF, PDGF, IGF-1, sérum) [19]. Les mécanismes par lesquels l'ET-1 conduit à la prolifération cellulaire incluent l'activation de la protéine kinase $\mathrm{C}$ (signal qui, s'il est nécessaire, ne suffit pas à lui seul à provoquer la prolifération cellulaire), l'autophosphorylation de $\mathrm{pp} 60^{\text {c-src }}$, ainsi que la stimulation de l'activité tyrosine kinase pp60 c-src [20], l'augmentation de la phosphorylation de certaines protéines cellulaires sur des résidus tyrosines (protéines dont l'identité n'est pas clairement définie), et l'activation prolongée de l'échangeur $\mathrm{Na}+/ \mathrm{H}^{+}[3]$. Tous ces événements conduisent secondairement à la stimulation par l'ET-1 de l'activité des MAP kinases [21], à l'induction de l'expression des protooncogènes $c$-fos et $c$-jun, provoquant ainsi la formation d'un complexe $\mathrm{AP}-1$, étapes qui précèdent généralement la prolifération cellulaire (pour une revue [19]).

Les endothélines exercent également de nombreux effets hépatiques. Nous avons caractérisé deux classes de récepteurs membranaires des endothélines, l'un liant l'ET-1 avec une meilleure affinité que l'ET-3 $\left(\mathrm{ET}_{\mathrm{A}}\right)$, l'autre liant l'ET-1 et l'ET-3 avec la même affinité $\left(\mathrm{ET}_{\mathrm{B}}\right)$. Les endothélines agissent dans le foie sur au moins trois types cellulaires. Dans l'hépatocyte, nous avons observé que l'ET-1 augmente de façon soutenue la glycogénolyse [22]. En accord avec ces données, Gandhi et al. [23] ont observé que l'ET-3 stimule la production de glucose dans le foie perfusé. L'ET-1 exerce son action glycogénolytique en augmentant le taux de calcium intracellulaire dans l'hépatocyte, cette élévation étant le résultat combiné de l'activation de la phospholipase $\mathrm{C}$, couplée à la protéine $\mathrm{Gq}$, et de l'inhibition de la pompe à calcium de la membrane 
Tableau II

LES EFFETS NON VASCULAIRES DES ENDOTHÉLINES

Cosur

Effets inotrope et chronotrope positifs

Effet biphasique (stimulation et inhibition) sur la sécrétion d'ANF

Effets hypertrophiques

\section{Cerveau}

Adénohypophyse : stimulation de la sécrétion de LH, FSH, TSH, GH, prolactine

Neuro-hypophyse : stimulation du relargage de vasopressine et oxytocine

Cervelet: stimulation du relargage de glutamate

\section{Effets mitogènes}

Stimulation dans les fibroblastes, cellules mésangiales, mélanocytes, cellules gliales, etc.

\section{Foie}

Activation de la glycogénolyse dans l'hépatocyte et de la sortie de glucose dans le foie perfusé

Modifications de la consommation d'oxygène hépatique

Stimulation de la production de PGE2 dans les cellules de Kupffer

Contraction des cellules de Ito

Augmentation de la pression portale

\section{Rein}

Inhibition de la sécrétion de rénine, diminution de la réabsorption de sodium, blocage de l'effet antidiurétique de la vasopressine

\section{Glandes surrénales}

\section{Stimulation de la sécrétion d'aldostérone}

LH : luteinizing hormone ; FSH : folliculostimulating hormone ; TSH : thyreostimulating hormone ; GH : growth hormone ; ANF : atrial natriuretic factor.

plasmique, couplée à Gs [13]. Les sarafotoxines se lient aux mêmes récepteurs que les endothélines et activent la glycogénolyse aux mêmes concentrations que les endothélines [24]. L'ET-1 ou l'ET-3 augmentent la pression portale et provoquent des modifications de la consommation d'oxygène hépatique [23]. Dans les cellules de Kupffer (macrophages résidents du foie), l'ET-3 stimule la phospholipase $\mathrm{C}$ par l'intermédiaire d'une protéine $\mathrm{Gi}$, et provoque la synthèse de prostaglandines (PGE2) [25]. Dans les cellules de Ito, qui jouent, entre autres, un rôle majeur dans la régulation du flux sanguin hépatique, l'endothéline-1 élève le taux de calcium cytosolique et induit la contraction [26, 27]. Enfin, la perfusion d'un foie avec un anticorps dirigé contre l'ET-1 bloque la vasoconstriction provoquée par l'éthanol, suggérant un rôle important de l'endothéline-1 dans les effets vasoconstricteurs provoqués par l'alcool au cours des cirrhoses [28].

Dans le cerveau, l'ET-1 est présente $\mathrm{m} / \mathrm{s} \mathrm{n}^{\circ} 10$ vol. 9 , octobre 93 et synthétisée dans les neurones du cordon médullaire et du ganglion de la racine dorsale ainsi que dans les cellules gliales, les astrocytes, les neurones hypothalamiques des noyaux supra-optiques et paraventriculaires. L'ET-3 serait la forme prédominante d'endothélines dans l'hypophyse. Les récepteurs des endothélines sont présents en grandes quantités dans le cerveau, et particulièrement dans le cervelet, le plexus choroïde, les noyaux des neurones supra-optiques et paraventriculaires et l'adénohypophyse [29]. Ils sont absents dans la neurohypophyse. Dans le système hypothalamo-hypophysaire, I'ET-1 stimule à la fois la sécrétion des hormones adénohypophysaires et neurohypophysaires. Ainsi, en se liant à des récepteurs spécifiques dans les cellules de l'adénohypophyse, l'ET-1 provoque une augmentation du calcium intracellulaire qui stimule la sécrétion des hormones gonadotropes (FSH et LH) de façon comparable à celle provoquée par le $\mathrm{GnRH}$, tout en se liant à des récepteurs distincts de ceux du GnRH [29]. Egalement, l'ET-1 stimule le relargage d'autres hormones adénohypophysaires, en particulier la TSH, l'hormone de croissance et la prolactine. D'autre part, l'ET-1 agit sur la neurohypophyse en provoquant le relargage de vasopressine et d'oxytocine. Enfin, l'ET-1 stimule le relargage de vasopressine et de substance $\mathrm{P}$ de tranches d'hypothalamus [29]. L'ET-3 active la sécrétion de $\mathrm{FSH}, \mathrm{LH}$ et TSH de cellules d'adénohypophyse en culture. De plus, l'ET-3 stimulerait ou inhiberait le relargage de prolactine de cellules d'adénohypophyse en culture. Ces résultats, apparemment contradictoires, s'expliqueraient par l'existence de deux types de récepteurs des endothélines dans ces cellules, relayant des effets différents. L'ensemble des données concernant l'ET-3 met en évidence le rôle majeur de ce peptide dans la régulation de la sécrétion des hormones hypophysaires [29]. Il faut noter que le rôle de neuropeptide des endothélines s'étend au cervelet et au cordon 
médullaire, où l'ET-1 stimule, respectivement, le relargage du glutamate et de la substance P [29].

Dans le cœur, les endothélines provoquent un effet inotrope positif très important et de longue durée, qui est sensible à la toxine de $B$. pertussis et relayé par une augmentation de calcium intracellulaire [30]. Le mécanisme conduisant à cet effet est peu clair et ne semble pas impliquer la phospholipase $\mathrm{C}$, dont l'activation par les endothélines est insensible à la toxine de $B$. pertussis [31]. En revanche, le blocage par la nicardipine de l'effet contractile provoqué par les endothélines suggère que l'activation des canaux calciques voltagedépendants de type $\mathrm{L}$ pourrait être impliquée [30], d'autant plus que la régulation de ces canaux par les endothélines est sensible à la toxine de $B$. pertussis [18]. Il est également possible que l'activation de l'échangeur $\mathrm{Na}^{+} / \mathrm{H}^{+}$par l'ET-1, partiellement sensible à la toxine de $B$. pertussis [30], et/ou l'inhibition du courant lent sortant de $\mathrm{K}^{+}[18]$, ayant pour conséquence la prolongation du potentiel d'action, participent à l'effet inotrope positif déclenché par les endothélines. Les endothélines exercent également un effet hypertrophique dans des myocytes isolés de rats nouveau-nés [32]. Cet effet se caractérise par une augmentation du volume des myocytes, une accumulation de protéines contractiles, l'induction des proto-oncogènes $c$-fos et $c$-myc et des gènes codant pour des protéines contractiles et embryonnaires. L'effet hypertrophique des endothélines serait sensible au $\mathrm{H}-7$, suggérant un rôle de la protéine kinase $\mathrm{C}$ dans cette réponse. Dans l'oreillette, les endothélines ont un effet chronotrope positif par un mécanisme mal élucidé. Enfin, l'ET-1 exerce un effet biphasique sur la synthèse et la secrétion de facteur natriurétique (ANF) [33]. A faibles doses (pM), l'ET-1 inhibe la secrétion d'ANF par un mécanisme impliquant les prostanoides [34]. A plus fortes doses (nM), l'ET-1 stimule la secrétion d'ANF par un mécanisme impliquant l'activation conjointe de la protéine kinase $\mathrm{C}$ et de la $\mathrm{Ca}^{2+}$ calmoduline kinase [34].

La liste des effets non vasculaires des endothélines s'allonge chaque jour, incluant des effets rénaux (inhibition de la sécrétion de rénine, diminution de la réabsorption de sodium, blocage de l'effet antidiurétique de la vasopressine), des effets sur les glandes surrénales (stimulation de la sécrétion d'aldostérone), des effets sur les muscles lisses non vasculaires (contraction du muscle intestinal, utérin...) $[3,4]$. Il faut souligner que, si des effets biologiques variés sont relayés par l'ET-1 dans différents tissus, en revanche le rôle de l'ET-3 serait limité au cerveau et les effets physiologiques de l'ET-2 sont inconnus.

\section{Les endothélines en pathologie}

Il est clair aujourd'hui que les endothélines exercent des effets biologiques variés relayés par au moins trois classes de récepteurs. Il reste encore de nombreux points à éclaircir, en particulier le rôle biologique de l'ET-2, la mise en évidence de nouveaux récepteurs qui sera certainement facilitée par le développement de nouveaux antagonistes, et la caractérisation des enzymes de conversion (en particulier l'ECE2 et l'ECE3). Mais une série de données nous montre l'importance que ces peptides pourraient avoir en pathologie. Ainsi, des taux élevés d'endothéline circulante ont été mesurés chez des patients atteints d'infarctus du myocarde, de cirrhose alcoolique, de septicémie ou d'insuffisance rénale [35]. D'autre part, l'injection intraveineuse du BQ123 à des rats les protège d'une insuffisance rénale aiguë et l'injection d'un nouvel antagoniste non peptidique et non sélectif (anti $\mathrm{ET}_{\mathrm{A}}$ et $\mathrm{ET}_{\mathrm{B}}$ ) bloque les effets vasoconstricteurs de l'ET-1 sur l'artère mésentérique et l'aorte [36]. La perfusion à des rats hypertendus de phosphoramidon, un inhibiteur de l'enzyme de conversion de la big ET-1, diminue considérablement leur pression artérielle [36]. Au cours de l'hypoxie alvéolaire pulmonaire, la concentration d'ET-1 est élevée, et après une hypoxie prolongée, l'activité vasoconstrictrice de l'ET-1 est augmentée alors que son effet vasodilatateur diminue très fortement, suggérant que l'ET-1 est impliquée dans l'hypertension pulmonaire hypoxique chronique. Ces données ont été récemment renforcées par la mise en évidence d'une augmentation de la sécrétion d'ET-1 et de l'expression de son ARN messager au cours de l'hypertension pulmonaire, de façon prédominante dans les cellules endothéliales vasculaires [37]. Mais c'est au cours de l'hémangio-endothéliome malin, une tumeur rare du cuir chevelu, que l'implication de l'ET-1 en pathologie est la plus probante. Chez ces patients qui présentent une prolifération intravasculaire maligne de l'endothélium, le taux d'ET-1 circulante est 20 fois supérieur à la normale et s'accompagne d'une hypertension. Ces deux paramètres reviennent à leur état de base lors de l'ablation de la tumeur, et s'élèvent à nouveau si la tumeur récidive. La concentration d'ET-1 dans la tumeur est 8 fois supérieure à celle du cuir chevelu, et s'accompagne d'une augmentation du taux d'ARN messager codant pour l'ET-1 dans le tissu tumoral [35]. Nous conclurons en soulignant que l'ET-1 pourrait également avoir une utilité thérapeutique puisque des données présentées au dernier congrès sur les endothélines (Houston, TX, USA, février 1993) suggèrent que l'ET-1 elle-même inverse l'hypotension consécutive au choc septique, à des doses pour lesquelles les fonctions rénales restent normales [36]

\section{TIRÉS A PART}

S. Lotersztajn. 


\section{Summary}

\section{Endothelins}

Endothelins are a family of three peptides of 21 amino acids, which cause a long lasting vasoconstriction preceded by an initial depressor response, when injected intravenously to rats. The three peptides (ET-1, ET-2 and ET-3) are encoded by three different genes and are derived from precursors ("big endothelins"), which are cleaved by a novel metalloprotease sensitive to phosphoramidon (endothelin-converting enzymes 1 , 2 or 3 ). ET-1 is a more potent vasoconstrictor than ET-3, while ET-1 and ET-3 are equipotent in inducing the depressor response, suggesting the existence of diffe- rent ET receptors. Indeed, two ET receptors have been cloned, the $\mathrm{ET}_{\mathrm{A}}$ receptor which binds ET-1 with a higher affinity than ET-3 and mediates vasoconstriction, and the $\mathrm{ET}_{\mathrm{B}}$ receptor which binds the endothelins with similar, high affinity and mediates vasodilatation. Also, recent data indicate that a subtype of $\mathrm{ET}_{\mathrm{B}}$ receptors would mediate some vasoconstrictor effects of endothelins and there are some evidences for the existence of a third class of $\mathrm{ET}$ receptor $\left(\mathrm{ET}_{\mathrm{C}}\right)$. The wide distribution of ET receptors, together with the presence of ET-1 mRNA in different tissues, suggest that ET may act as local hormones. Accordingly, in addition to their role in vascular tissues, endothelins have been shown to cause non vascular effects in heart, kidney, adrenal glands, liver, and to act as neuropeptides and growth factors. Endothelins exert their effects by increasing cytosolic free calcium consecutively to stimulation of phospholipase $\mathrm{C}$ and calcium channels, and inhibition of the plasma membrane calcium pump. Endothelins also activate protein kinase $\mathrm{C}$, raise or decrease cAMP levels, stimulate phosphatidylcholine hydrolysis, phospholipase A2, $\mathrm{Na}+/ \mathrm{H}^{+}$exchange, modulate ionic channels and phosphorylate cytosolic proteins on tyrosine residues. The role of these peptides in pathophysiology is emerging, and should be resolved with the development of receptor antagonists and ECE inhibitors. 